\title{
Reflexiones del uso del teatro como herramienta didáctica para la enseñanza de lenguas extranjeras: Entre beneficios y ventajas
}

\section{Reflections on the use of theater as a educational tool for teaching foreign languages: benefits and advantages}

Derly Cervantes Cerra ${ }^{1}$

Universidad del Atlántico

\section{RESUMEN}

El presente artículo de reflexión tuvo como objetivo el analizar los distintos beneficios

1 Orcid: 0000-0001-5386-2497

Dirección: Calle 19 \#22-24 Barrio Centro de Soledad

Correo electrónico: derly_cervantes@hotmail.com

Títulos académicos: Magíster en francés como Lengua Extranjera

Afiliación institucional: Docente tiempo completo ocasional en el departamento de Lenguas Extranjeras en la Universidad del Atlántico, Colombia.

Universidad del Atlántico: ubicada en kilómetro 5, vía Puerto Colombia, Teléfono: (035) 3853002 y ventajas que el uso del teatro tiene en la enseñanza de lenguas extranjeras. Para ello, se realizó una revisión documental en la que se enfocó la relación del teatro con algunos enfoques como el enfoque comunicativo y el de tareas, que son dos de los más empleados en la enseñanza de L2. Asimismo, se basó en autores como Corral (2013), Casas (2015), Palomo (2019), entre otros. Igualmente, fue un trabajo enmarcado en el paradigma cualitativo siendo así de tipo revisión documental- descriptivo. Entre sus resultados destacó que el uso del teatro como herramienta didáctica en el aula 
de L2 favorece el desarrollo de habilidades y competencias comunicativas, en especial de la expresión y comprensión oral. Se afirma, además, que este tema requiere de un mayor acercamiento e investigación por parte de la academia.

PALABRAS CLAVES: lenguas extranjeras, teatro, aprendizaje.

\section{REFLECTIONS ON THE USE OF THEATER AS A EDUCATIONAL TOOL FOR TEACHING FOREIGN LANGUAGES: BENEFITS AND ADVANTAGES}

\section{ABSTRACT}

The aim of this article was to analyze the different benefits and advantages that theatre has in the foreign languages teaching. In order to do this, a documentary review was conducted focusing on the relationship of the theatre with some important perspectives. For instance, the communicative approach and the task-based approach, which are two of the most used in the teaching of L2. Likewise, it also relied on authors such as Corral (2013), Casas (2015), Palomo (2019), among others. Similarly, it was a work framed in the qualitative paradigm embedded in a documentary-descriptive revision. Among the most significant findings, he emphasized that the use of theatre as a didactic tool in the L2 classroom, it enhances the development of communication skills and competences; in particular, the expression and oral comprehension. In addition, it is affirmed that this topic requires a greater approach and investigation by the academy.

KEYWORDS: foreign languages, theater, learning.

\section{INTRODUCCIÓN}

Para nadie es un secreto que el aprender una segunda lengua no es una tarea sencilla. Porque este es un proceso que involucra factores internos y externos, como "la motivación, las técnicas de enseñanza, los estilos de aprendizaje, el ambiente en que se aprende hasta los materiales didácticos que se usan, el enfoque educativo, entre otros" (Salas, 2019, p. 100).

Ahora bien, como docente de lenguas extranjeras es necesario tomar en cuenta todos estos elementos a la hora de diseñar las estrategias de enseñanza que le faciliten a los estudiantes su proceso de adquisición de L2 (Del Valle Bierbrauer, 2013; Di Pietro 1987; Dorado, 2016; Drew, 2013).

En este sentido, para algunos autores como Alonso (1990), Gabino (2014), Flores (2016), Hidalgo y Tobal (2014) entre otros, el contexto es uno de los elementos de mayor relevancia en el diseño de estrategias porque le permite al docente entender las necesidades e intereses de los estudiantes.

Igualmente, dentro de la adquisición de una lengua existen competencias comunicativas que debe desarrollar el estudiante como la escritura, la lectura, la expresión oral y la escucha. Asimismo, debe comprender el aspecto pragmático subyacente en los actos comunicativos. Y es que según Ussa como fue citado en Salas (2019) en el aprendizaje de una L2 "es indispensable tener en cuenta el contexto social en donde se desarrolla la actividad lingüística" (p. 109).

Es decir, el estudiante no solo debe manejar las reglas morfosintácticas, el valor semántico y otros aspectos como el fonológico, entre otros. Debe, además, conocer las distintas intenciones de los hablantes en función de los contextos en el que se da la comunicación.

En este orden, una de las estrategias más empleadas en la enseñanza de lenguas que involucra las cuatro competencias comunicativas ya mencionadas y el entendimiento del contexto 
comunicacional, es el teatro. Entendiéndose que "cuando hablamos de teatro nos referimos a una representación teatral que se escenifica y que tiene un público pasivo" (Corral, 2013, p. 21)

El teatro, además, ha estado presente desde varios milenios en la historia de la humanidad, ya sea con fines meramente recreativos como en la época de la antigua Grecia, por ejemplo, o con fines de adoctrinamiento ético - moral como ocurría en la llamada época dorada del teatro europeo en los siglos XVI y XVII (Lastra, 2017; Odriozola, 2015; Peña, 2018) Esta valiosa herramienta ha estado presente en el aula de lenguas extranjeras desde hace varios siglos. Por eso, en el presente trabajo se analizarán grosso modo, las ventajas y beneficios de su uso en la enseñanza de lenguas extranjeras. $Y$ es que el teatro se ha hecho popular en la enseñanza de L2 porque en la escenificación de una obra los estudiantes pueden practicar desde su pronunciación, pasando por la práctica de la escucha y por supuesto, recreando actos comunicativos que atienden a un grupo de factores cuyo valor semántico está estrechamente vinculado a la lengua que se está aprendiendo.

Esto se afirma partiendo del siguiente fragmento "representan uno de los grandes pilares sobre los que se apoya la enseñanza y práctica de la expresión oral" ya que "requieren por parte del alumno la creación y escenificación de una situación comunicativa determinada" (Corral, 2013, p. 123).

\section{ENFOQUE POR COMPETENCIA Y TAREAS EN EL TEATRO}

Antes de ver la relación entre estos dos enfoques y el teatro, es necesario definirlos grosso modo. En primer lugar, el enfoque por competencias comunicativas se define según Gil (2019) como aquel que:

centra su dinámica de enseñanza en enunciados que, si reflejan situaciones comunicaciones reales, para ello diseñan recursos y materiales que recrean actividades que propician la interacción auténtica entre los estudiantes y sus compañeros en pro de que practiquen lo aprendido de la Lengua extranjera (p. 174)

En otros términos, el enfoque por competencia hacia énfasis en el desarrollo y fortalecimiento de las habilidades y destrezas comunicativas de los estudiantes mediante la recreación de situaciones que emulan a la comunicación en contextos reales.

De igual forma, el enfoque por tareas "es un enfoque que motiva al estudiante a avanzar poco a poco en su aprendizaje de LE por medio de ejercicios adecuados a contenidos, pautas y objetivos en específicos." (Gil, 2019, p. 177). Es decir, que en este se realizan tareas o actividades que incluyen la práctica de distintas habilidades en función de lograr objetivos determinados.

Asimismo, Palomo (2016) explica con respecto a la tarea lo siguiente:

La tarea reviste la forma de proyectos finales de unidad, en general grupales, que abarcan contenidos tratados recientemente en clase y que buscan un "refresco" y consolidación de todo el bagaje comunicativo adquirido en un determinado lapso de tiempo. Todas las destrezas comunicativas se ejercitan en el enfoque por tareas (p. 199)

Ahora bien, el teatro como herramienta didáctica en el aula de lenguas extranjeras fusiona elementos de ambos enfoques, como por ejemplo la recreación de situaciones comunicativas reales, la práctica de competencias comunicativas como el habla, la escucha, la lectura e incluso la escritura. 
Asimismo, el teatro según Flores (2016) "Se integra perfectamente en los presupuestos del enfoque comunicativo y, en particular, los autores lo proponen como ideal dentro del enfoque por tareas, ya que la representación es un cierre sumamente apropiado como tarea final". (p. 6).

\section{USO DEL TEATRO EN EL AULA DE LENGUAS EXTRANJERAS}

Como ya se ha mencionado, la utilización del teatro en la enseñanza de lenguas extranjeras no es algo del todo nuevo, sin embargo, el enfoque por tareas lo incluye como una estrategia multimodal porque permite la "sistematización e integración de la gramática, del léxico, o el trabajo sobre la pronunciación y entonación siempre en armonía con el cuerpo" (Corral, 2013, p. 120).

Dicho de otra forma, es una estrategia sumamente completa porque estimula el ejercicio y la práctica de distintas habilidades y competencias comunicativas. De igual manera, el teatro va más allá del aspecto académico, llega hasta incluso lo afectivo porque promueve la motivación y creatividad en el educando y es que según Casas (2015) "podemos utilizar el teatro (ensayos y representación escénica) como técnica de aprendizaje (...) para motivar y enseñar", siempre que hablemos de teatro como un proceso continuo de aprendizaje (p. 6).

En otras palabras, el teatro estimula el interés de los estudiantes en su proceso de aprendizaje mientras le ayuda a combinar los conocimientos adquiridos en recreaciones de situaciones comunicacionales.

Asimismo, la motivación es sin duda alguna, un factor sumamente importante a la hora de enfocar el proceso de enseñanza-aprendizaje, porque un estudiante motivado tendrá una mejor disposición para la realización de las actividades que uno que no lo está. $Y$ es que:
El intelecto rara vez funciona sin un elemento emotivo que muchas veces falta en los materiales didácticos convencionales. Sin emoción, el aprendizaje se ralentiza, todo se vuelve más mecánico y rutinario. El alumno ha de poder desarrollar las habilidades 0 destrezas necesarias que están presentes en todos los actos comunicativos de cada una de las lenguas para poder ser competente comunicativamente (anticipación, rapidez de reacción, perspicacia, adaptabilidad, sensibilidad en el tono, etc.) (Casas, 2015, p. 6)

Dicho de otro modo, el teatro representa una forma de romper con la rutina tradicional del aula, en el que el estudiante se involucra de forma más activa en su propio proceso de enseñanza. Esto es sin duda, un valor agregado a la dinámica del aula de lenguas extranjeras porque promueve y estimula el interés de los educandos en las clases.

Otro beneficio al aspecto afectivo, es que el teatro fortalece la autoestima del estudiante y lo invita a empoderarse de su proceso de aprendizaje porque le permite experimentar una situación en la que es el "auténtico protagonista del proceso educativo, en un ambiente donde las habilidades personales de cada individuo son complementarias y responsables de la tarea grupal, y donde los errores cometidos se difuminan o se reciclan en creatividad y dinamismo" (Palomo, 2016, p. 200). Es evidente, que también promueve el aprendizaje colaborativo mejorando así, la interacción entre estudiantes y estudiantes.

Con respecto a otras ventajas del uso del teatro en el aula de lenguas extranjeras, Maley como fue citado en Casas (2015) agrega "La explotación del teatro nos puede ayudar a la enseñanza del significado gracias al uso de la lengua en contexto, sin importar lo fantástico que pueda llegar a ser (p. 6). Es decir, que independientemente del género de la obra que 
se escenifique esta tendrá grandes beneficios en cuanto a la asimilación de la carga semántica de las palabras y de los diálogos que se representen en sí.

Otras ventajas del teatro en el aula de lenguas extranjeras, son los diferentes juegos y actividades que se derivan de este. Al respecto, Palomo (2016) expone:

- juego de personajes (actividad más sencilla, con más improvisación, más práctica de las destrezas orales, más control de los elementos gramaticales y comunicativos)

- juego de simulación (más compleja, necesidad de mayor preparación, integradora de destrezas)

- juego de dramatización, esto es, dotar de contenido dramático (más bien, de forma dramática) a un texto literario, ya sea una narración, poema, noticia, canción, etc. ( $p$. 211)

Es evidente, el teatro representa una gran gama de opciones en cuanto a actividades didácticas se refiere. Porque el docente, puede fusionar otras tareas con la escenificación ya sea de diálogos o situaciones.

Igualmente, tiene excelentes beneficios en la oralidad puesto que Flores (2016) expone lo siguiente:

a) Favorece primordialmente la expresión oral: mejora la pronunciación, la entonación, la acentuación, etc. Es ideal para desarrollar mayor fluidez en los estudiantes. Todos los aspectos suprasegmentales, tan difíciles de incluir normalmente en las clases, pueden ser explotados a partir del teatro. b) Facilita la adquisición de vocabulario y refuerza las estructuras lingüísticas aprendidas previamente en clase. (p. 6)
Es palpable en el fragmento, que el teatro conjuga habilidades que tradicionalmente no son del todo incluidas en las actividades cotidianas del aula de lenguas extranjeras. Lo relevante acá es que lo hace de forma simultánea puesto que mientras el estudiante escenifica practica varias habilidades que incluyen oralidad, escucha, léxico, entre otras.

Destaca en especial, la expresión oral como habilidad que se ejercita en el teatro y que como es bien conocido, es una de las destrezas que más dificultades les trae a los estudiantes.

Esto se debe a que el aprendizaje verbal:

ocurre en virtud de la asimilación de la palabra u otro tipo de actividad simbólica, ya sea como estímulos externos o como contenido de la conciencia humana. El aprendizaje verbal aporta al individuo la experiencia social, la cultura de la sociedad elaborada históricamente y engendra por lo general de manera consciente los conocimientos, habilidades y capacidades, las intenciones, proyectos, sentimientos y necesidades superiores, o sea, produce el psiquismo específicamente humano, la personalidad (González, 2015, p. 127)

Es decir, que cuando el estudiante realiza actividades que incluyen la expresión oral como la escenificación en teatro, por ejemplo, realmente ocurre un aprendizaje significativo porque este tipo de ejercicios incluye múltiples factores como el entendimiento del contexto, el valor pragmático del acto comunicativo, entre otros.

\section{CONSIDERACIONES GENERALES}

A lo largo de este trabajo se han destacado solo algunos de los muchísimos beneficios y ventajas que tiene el uso del teatro como herramienta didáctica en la enseñanza de lenguas extranjeras, como, por ejemplo, sus cualidades como herramienta versátil, 
innovadora e incluso, motivacional, así como las ventajas que tiene su uso en el desarrollo de las habilidades y competencias comunicativas como la producción y compresión oral, el mejoramiento del vocabulario, entre otras. (Alonso, 1990; Berto, 2018)

Asimismo, el valor como herramienta motivacional parece ser uno de sus puntos más fuertes porque representa una actividad que se distingue de la mayoría de los ejercicios que se realizan tradicionalmente en el aula de L2. Sin dejar de lado, que su práctica requiere de la utilización de varias habilidades y destrezas en la segunda lengua, que pese a ser un reto para los estudiantes, resulta ser un ejercicio enriquecedor y ameno.

El teatro como herramienta didáctica promueve, además, el aprendizaje colaborativo porque incita a los estudiantes a apoyarse mutuamente y salvaguardar de forma indirecta, las diferencias y vicisitudes. Sin duda alguna, el uso del teatro en la clase de L2 tiene muchos beneficios que ofrecer tanto a los docentes como a los estudiantes, lo que evidentemente invita a su investigación de forma más detallada en futuros estudios.

\section{REFERENCIAS BIBLIOGRÁFICAS}

Alonso, R. (1990). Enseñanza interactiva del español a través de escenarios. Aplicaciones didácticas. ASELE, actas II: 209-216.

Berto, A. (2018) La motivación y la interacción entran en escena el teatro: un método dinámico para aprender español como lengua extranjera y lengua materna (Tesis de Maestría). Universitat Jaume I. Castellón de la Plana, España.
Casas, I. (2015) Uso del teatro en el aula de lenguas extranjeras adaptación de Matilda como propuesta didáctica (Tesis de Maestría). Universidad de Almería. Almería, España.

Corral, A. (2013). El teatro en la enseñanza de lenguas extranjeras. La dramatización como modeloy acción. Didáctica. Lengua y Literatura, 25, 117-134. Recuperado de http://revistas.ucm.es/index.php/ DIDA/article/view/42238/40212

Del Valle Bierbrauer, Fanny (2013) Estudio de estrategias en juegos de rol en situación de examen Caso Certificado de Español Lengua y Uso. Universidad de Córdoba. Trabajo fin de Máster.

Di Pietro, R. (1987). Strategic Interaction: Learning Languages through Scenarios. Cambridge: Cambridge University Press

Dorado, M. (2016). La implementación de las bellas artes en la enseñanza de segundas lenguas (Tesis de maestría en artes) Colorado State University, Colorado, Estados Unidos. Recuperado de https://mountainscholar.org/ bitstream/handle/10217/176607/ DoradoBudia_colostate_0053N_13635. pdf?sequence $=1$

Drew, I. (2013). "Linking theatre to CLIL in foreign language education". Nordic Journal of Modern Language Methodology, vol. 2, 1. Recuperado de http://journal.uia. no/index.php/NJMLM/article/view/69\#. $\underline{\mathrm{U} 5 \mathrm{r} 8 \mathrm{y} 3 \mathrm{a} 2 \mathrm{fXQ}}$

Flores, M. (2016). El teatro en la enseñanza de ELE/EL2: un estado de la cuestión. VI Jornadas de Español como Lengua Segunda y Extranjera, 21 y 22 de octubre de 2016, Ensenada, Argentina. Reflexiones sobre el examen CELU (Certificado de Español 
Lengua y Uso). EN: Actas. Ensenada : Universidad Nacional de La Plata. Facultad de Humanidades y Ciencias de la Educación. Especialización en la Enseñanza del Español como Lengua Extranjera. En Memoria Académica. Recuperado de http://www.memoria. fahce.unlp.edu.ar/trab_eventos/ ev.10022/ev.10022.pdf

Gabino, M. (2014) "Interacción y motivación en el aula: enseñar desde el teatro", en Tobar Delgado, E. e Hidalgo Martín, $\checkmark$ (coords.). Arriba el telón: enseñar Teatro y enseñar desde el teatro. Mihno: SECRETARÍA GENERAL TÉCNICA: 5-20.

Gil, N. (2019) El enfoque por tareas en la enseñanza de lenguas extranjeras: reflexiones de su origen y relación con otros enfoques. Boletín REDIPE, 8 (9), 170-81. Recuperado de https://revista. redipe.org/index.php/1/article/view/820

González, D. (2015). Una concepción integradora delaprendizajehumano.PERSPECTIVA, Florianópolis, 33 (1), 119-134. DOI: http://dx.doi. org/10.5007/2175795X.2015v33n1p119

Hidalgo, V. y Tobal, E. (eds.) (2014). Arriba el telón: enseñar teatro y enseñar desde el teatro. Propuestas didácticas para enseñar teatro en clases de español. Mihno: SECRETARÍA GENERAL TÉCNICA

Lastra, C. (2017). The benefits of arts education in second language acquisition and in pedagogy. (Tesis de maestría en educación en arte). Humboldt State University, California, Estados Unidos. Recuperado de https:// digitalcommons. humboldt.edu/cgi/viewcontent. cgi?article $=1055 \&$ context $=$ etd
Odriozola, M. (2015) El teatro como herramienta para el aprendizaje del inglés: una propuesta de intervención (Tesis de Pregrado). Universidad Internacional de la Rioja. La Rioja, España.

Palomo, F. (2016) El teatro y sus aplicaciones didácticas en la enseñanza del FLE. Claves pedagógicas, sociales y lingüísticas. Balance de una experiencia llevada a cabo en Educación Secundaria (Tesis doctoral). Universidad de Granada. Granada, España.

Peña, D. (2018). La pedagogía teatral en el desarrollo de los imaginarios socioculturales infantiles. Colegio Los Andes de Bogotá. Boletín REDIPE, 7(10), 166-77. Recuperado de https:// revista.redipe.org/index.php/1/article/ view/591

Salas, J. (2019) Las artes escénicas en el aprendizaje de una segunda lengua: la experiencia del proyecto ED-3091: fortalecimiento de la enseñanza y el aprendizaje del inglés. Revista Pensamiento Actual, 19 (32), 99. - 114 doi 10.15517/PA.V19132.37879 